\title{
Postindustrielle oder industrielle Dienst- leistungsgesellschaft: Wohin treibt die gesellschaftliche Arbeitsteilung?
}

\begin{abstract}
Mit den Kräfteverschiebungen der Weltwirtschaft und dem Abbau politischer Regulierungen verschieben sich beschleunigt auch die Proportionen zwischen gering und hochqualifizierter, industrieller und tertiärer, männlicher und weiblicher Arbeit und zwischen privilegierten und prekären Arbeitsverhältnissen. Damit sind auch die bisherigen „Zeitdiagnosen“ herausgefordert, die ein unaufhaltsames Ende der Industriegesellschaft und ihre Ersetzung durch eine Dienstleistungs- oder Wissensgesellschaft oder auch ihre Auflösung in eine zusammenhanglose Vielfalt prophezeit haben. Lineare Trendprognosen und pauschalisierende Dateninterpretationen werden der komplexen, widersprüchlichen Realität der Arbeitsteilung nicht gerecht.
\end{abstract}

\section{Das Doppelgesicht der Arbeitsteilung}

Die reale Erwerbsstruktur ist ein komplexes Mosaik von arbeitsteilig spezialisierten und komplementären Funktionen, das durch seine Dynamik eher einem Mobile gleicht, in dem viele ungleiche und veränderliche Teile aufeinander wirken und voneinander abhängen. Die Entwicklung unterliegt daher auch nicht alternativlosen Einheitstendenzen. Das soziale Gefüge, als bewegtes Feld konkurrierender Kräfte und kämpfender Akteursgruppen, ist in seinen Entwicklungen zwar nicht völlig berechenbar. Gleichwohl hat es seine eigenen Logiken, deren Kenntnis den wahrscheinlichen Spielraum möglicher Alternativen und Trendwenden durchaus voraussehbar machen kann.

Die Wissenschaft tut sich damit allerdings schwer. Mangels eines Gesamtkonzeptes der Analyse beschränkt sie sich meist auf Datenreihen über Einzelentwicklungen. Damit löst sie aber die Einzeldynamiken aus dem realen Zusammenhang ihrer Bedingungen und Gegendynamiken und erleichtert es, sie zu linearen Einheitstrends zu verabsolutieren.

Angesichts der heutigen Datenfülle ist es vielversprechend, das Mosaikbild der Realität zu ordnen und die komplexen Logiken der Entwicklungen aufzudecken.
Dafür geeignet ist immer noch das klassische Konzept der gesellschaftlichen Arbeitsteilung, das von der Differenzierung in spezialisierte und komplementäre Berufe und Berufsgruppen ausgeht. Die klassischen Wirtschafts- und Gesellschaftstheorien - von Smith und Ricardo bis Marx und Durkheim - haben dieses Konzept genutzt, um den Gesamtzusammenhang und die Dynamik der gesellschaftlichen Entwicklung zu untersuchen und zu erklären.

Das Problem ist, dass diese Theorien oft in einseitiger und verkürzter Form wiedergegeben und als Waffe in ideologischen Kämpfen benutzt werden. Vulgärmarxistische Theorien betonen die Klassenpolarisierung kapitalistischer Gesellschaften und die Tendenzen zur Fragmentierung bzw. Taylorisierung der Arbeit, zur Entfremdung des Arbeiterbewusstseins und zur Homogenisierung und Herabdrückung der sozialen Lagen. Spiegelbildlich dazu sehen vulgärliberale Theorien der „Modernisierung" die Entwicklung als eindimensionale Erhöhung der wirtschaftlichen Effizienz, der Produktivität und des allgemeinen Wohlstands durch Differenzierung.

Demgegenüber verstehen die klassischen Theorien soziale Ungleichheit und Produktivitätserhöhung als Teile des gleichen Strukturwiderspruchs. Sie heben hervor, dass in kapitalistischen Gesellschaften das ökonomische System nicht allein der Herrschaftsausübung und der Gewinnerhöhung dient, wie in vorangehenden Gesellschaften. Es muss auch die
Produktivkräfte der Arbeit und Technik und die Herstellung von Gebrauchswerten immer weiter entwickeln. Durch diesen Widerspruch bringt es von sich aus auch Potenziale des Wandels hervor.

Von allen Autoren hat Emile Durkheim (1988) am deutlichsten herausgearbeitet, dass es dabei um einen Widerspruch zwischen emanzipatorischen und herrschaftsorientierten Kräften geht.

Nach Durkheims Diagnose wachsen die Potenziale der Emanzipation, der mündigen Selbstbestimmung, weil mit der beruflichen Spezialisierung nicht nur die Arbeitsproduktivität und die ökonomische Interdependenz zunehmen, sondern auch höhere Niveaus von Bildung, Kompetenz und Kooperation entstehen. Es bleibt in der Regel nicht aus, dass die Menschen in den Berufsmilieus auch gemeinsame moralische Regeln, ein Berufsethos und einen gemeinsamen „moralischen Habitus" entwickeln und durch fachliche Spezialisierung gleichzeitig auch Individualität hervorbringen, dass sie auf dieser Grundlage Interessengruppen bilden und um politische Repräsentation kämpfen (ebd., S. 44, 55f., 259f., 474f.).

\footnotetext{
Michael Vester, Dr., Prof. i. R. an der Leibniz Universität Hannover. Leiter der Forschungsgruppe Habitus und Milieu.

Arbeitsschwerpunkte: Politische Soziologie und Geschichte sozialer Strukturen, Mentalitäten, Bewegungen und Partizipation. e-mail: m.vester@ipw.uni-hannover.de
} 
Diese emanzipatorischen Möglichkeiten werden jedoch, so Durkheim, eingeschränkt oder vereitelt, wenn an die Stelle der von den Berufsmilieus selbst hervorgebrachten Arbeitsteilung, die auf „Konsensus" und "Solidarität" beruht, eine durch Herrschaftshierarchien „erzwungene Arbeitsteilung“tritt, in der „die Funktionen derart verteilt sind, dass sie dem Individuum nicht genügend Raum zum Handeln bieten. “Wenn das „abgestimmte Verhältnis zwischen den Fähigkeiten der Individuen und der Art der ihnen zugewiesenen Tätigkeit gestört" werde, seien „schmerzhafte Reibungen“ und „Klassenkämpfe“ die Folge (ebd., S. 443ff., 459).

Als Lösung dieses Problems schlug Durkheim, der sich auch stark in der Arbeiterbildung engagierte, eine in den Berufsgruppen beginnende demokratische Selbstund Mitbestimmung vor (ebd., S. 70). Er verdeutlichte dies, mit Blick auf die damals entstehende Arbeiterbewegung, mit einer historischen Parallele: „Als die Plebejer den Patriziern die Ehre der religiösen und administrativen Funktionen streitig machten, taten sie dies nicht einfach zu deren Nachahmung, sondern weil sie intelligenter, reicher, zahlreicher geworden waren und sich infolgedessen ihr Geschmack und ihr Ehrgeiz geändert hatten.“ (ebd., S. 444f.)

\section{Die politische Modifikation der Berufsgliederung}

Die Berufsgruppen, als Grundeinheiten der gesellschaftlichen Arbeitsteilung, treten in der klassischen Theorie in doppelter Gestalt auf: im ökonomischen Feld als wirtschaftliche Produktivkräfte, im gesellschaftlichen Feld als Akteure sozialen Handelns, d.h. der Identitätsbildung und der Interessenpolitik nicht zuletzt der Arbeitnehmer und Unternehmer. Über ihre Zusammenschlüsse, über das korporative Aushandlungssystem und über die staatliche Sozial- und Wirtschaftspolitik können sie auf die Gestaltung der ökonomischen Arbeitsteilung zurückwirken.

Die Berufsgliederung kann durch politische Regulierungen modifizierend beeinflusst werden, auch wenn sie in ihren Grundmustern primär von den Prozessen im nationalen und internationalen ökonomischen Feld abhängt. Selbst in Ländern mit gleichem ökonomischen
Entwicklungsstand können die Proportionen zwischen den Berufsgruppen und die Anteile der Geschlechts-, Alters- und Ethnogruppen in ihnen bis zu einem gewissen Grade verschieden sein. Dies hängt davon ab, welchem Pfad der Regulierung die Politik eines Landes folgt.

Der skandinavisch-sozialdemokratische Wohlfahrtsstaat ist trotz seiner Unzulänglichkeiten bis heute das Referenzmodell für einen Pfad geblieben, der gleichzeitig den sozialen Ausgleich und die Dynamik der ökonomischen Produktivkräfte fördern kann. Er erhöhte die Beschäftigung im öffentlichen Gesundheits-, Bildungs- und Sozialsektor und den Aufstieg in qualifizierte technische und soziale Berufe. Damit förderte er gleichzeitig die Berufsfelder nicht zuletzt im öffentlichen Dienst, in denen die Erwerbschancen der Frauen traditionell größer waren. Im konservativen deutschen Modell des Wohlfahrtsstaates geschah dies deutlich gebremster, im liberalen angelsächsischen Modell in nur geringem Maße.

Die klassischen Unterschiede zwischen sozialdemokratischen, konservativen und liberalen Pfaden, die sich nach dem Zweiten Weltkrieg entwickelt haben, sind allerdings seit den 1970er Jahren durch neue internationale Deregulierungen und durch die Gewichtsverschiebungen im weltwirtschaftlichen Kräftefeld ins Rutschen gekommen. Unter deren Druck und durch politische Pfadänderungen kam es in vielen Ländern zu neuen Verschiebungen zwischen den Berufsgruppen und zu neuen Polarisierungen zwischen privilegierten und benachteiligten sozialen Lagen. Seit der jüngsten Wirtschaftskrise setzen sich die zunehmenden sozialen Spannungen auch in neue Protestbewegungen um.

\section{Die Herausforderung durch neue Dynamiken}

Die mächtigen Kräfteverschiebungen, die die Wohlfahrtsstaaten seit den 1970er Jahren zunehmend herausfordern, können auf beiden Achsen der widersprüchlichen Struktur der gesellschaftlichen Arbeitsteilung verortet werden. Zum einen hielt die Differenzierungsdynamik der Produktivkräfte an, zum anderen geriet sie zunehmend unter das Vorzeichen neuer sozialer Polarisierungen.
Die unter den Wohlfahrtsregimes bereits beschleunigte Dynamik der menschlichen und technischen Produktivkräfte wurde, unter dem zunehmenden Druck der internationalen Konkurrenz, in der neuen, informationstechnologischen Revolution noch einmal ungeahnt gesteigert. In Politik und Wissenschaft wurden vor allem drei tief greifende Wandlungen des Sozialgefüges lange diskutiert:

- die Zunahme höherer Berufsqualifikationen und Bildungsabschlüsse (Kompetenzrevolution),

- die nachhaltige Expansion der Dienstleistungsberufe (Tertiarisierung),

- das erhebliche Wachstum der Erwerbstätigkeit von Frauen (Feminisierung).

Die vielfältigen Diskussionen der drei Dynamiken, die hier nicht im Einzelnen wiedergegeben werden können, verliefen nicht zuletzt deswegen ergebnislos, weil sie die neuen Erscheinungen nicht durch eine zusammenhängende Theorie oder Analyse erklären konnten. Die drei Dynamiken wurden vorwiegend als lineare Einzeltendenzen absolut gesetzt - und gerade deswegen auch äußerst kontrovers debattiert. Zur ersten Dynamik standen Thesen einer Wissens- oder Bildungsgesellschaft und des Aufstiegs einer neuen akademischen Elite zur Diskussion. Zur zweiten Dynamik wurden die optimistischen Theorien der Tertiarisierung teilweise bis zur These eines Endes der Industriegesellschaft und der Durchsetzung einer „postindustriellen Dienstleistungsgesellschaft" gesteigert, in der die Klassenunterschiede verschwinden würden. Im Zusammenhang damit wurde die dritte Dynamik als Ersetzung der Klassen durch Differenzierungen nach Geschlecht diskutiert, teilweise in Verbindung mit Differenzierungen nach anderen, insbesondere ethnischen Zugehörigkeiten.

Mit dem politischen Pfadwechsel änderten sich jedoch die positiven Vorzeichen dieser Entwicklung. Im Kontext des ausgleichenden Wohlfahrtsstaats hatten die drei Dynamiken die sozialen Ungleichheiten zwar nicht nivelliert, aber doch dazu beigetragen, die frühere steile Pyramide der Ungleichheit durch eine Sozialstruktur mit geringeren Ungleichheiten und einer breiten Mitte zu ersetzen. Die neoliberale Deregulierung der inneren und der internationalen Beziehungen wirkte dagegen widersprüchlich. Der Leistungswettbewerb verstärkte die Differenzierung der 
Erwerbsstruktur und die Produktion des gesellschaftlichen Reichtums. Zugleich wurde aber eine Umverteilung dieses wachsenden Reichtums nach oben eingeleitet.

Auch in Deutschland wurden die Exporterfolge mit Kostensenkungen erkauft, durch die die Leistungen des Sozialstaats, die Arbeitseinkommen und damit die inländische Nachfrage und mit dieser auch die Importquote gesenkt wurden. Die Einkommen in der sozialen Mitte stagnierten seit den 1980er Jahren und wurden später unsicherer. Im untersten Fünftel der Gesellschaft kehrten zunehmend unterprivilegierte Einkommens- und Soziallagen zurück. Die Verteilung sozialer Chancen wurde ungleicher durch die Bremsung der Bildungsexpansion, des Ausbaus und der Leistungen der öffentlichen Bildungs-, Gesundheits- und Sozialdienstleistungen und der gleichen Erwerbschancen von Frauen.

Mit den sozialen Polarisierungen haben inzwischen die wirtschaftlichen Ungleichgewichte innerhalb wie zwischen den einzelnen Ländern so zugenommen, dass eine Erholung aus der Wirtschaftskrise ohne ein politisches Umsteuern und institutionelle Reformen unmöglich erscheint. Die Eurokrise beruht, wie Helmut Schmidt jüngst wieder hervorhob (Frankfurter Rundschau, 26.8.2011, S. 11), auf den gigantischen Überschüssen der deutschen Leistungsbilanz. Das sei „vielen anderen europäischen Regierungen und auch der öffentlichen Meinung in diesen Ländern zu Recht ein Dorn im Auge." Abhilfe verschaffen könne nur eine Belebung der Binnennachfrage in Deutschland. Der keynesianische Nobelpreisträger Joseph Stiglitz verlangte in Madrid vor Angehörigen der neuen Demokratiebewegungen eine wirtschaftspolitische Kehrtwende und eine entschiedene Stärkung der Inlandsnachfrage durch höhere Einkommen sowie Investitionen in Bildung, Spitzentechnologien, Dienstleistungen und Infrastrukturen (El País, 26.7.2011, S. 1, 14). Das heißt, dass nicht nur die finanzpolitischen Regulierungen, sondern auch die Arbeitsteilungen innerhalb der einzelnen Volkswirtschaften und zwischen ihnen neu justiert und ausbalanciert werden müssen.

Damit stehen Konflikte über die Frage an, was die deutsche Politik tun kann, um im Gefüge der Arbeitsteilung das $\mathrm{Hu}-$ mankapital bzw. die höher qualifizierten Gruppen (nicht zuletzt der Ingenieure und technischen Experten) und der wohlfahrtsstaatlichen Dienstleistungen (Bildung, Gesundheit, Kultur, Soziales und vor allem kommunale Infrastrukturen) stärker zu fördern und auszubauen. ${ }^{1}$ Es geht dabei um ein Bündel verkoppelter Probleme langfristiger Strukturentwicklungen:

(1) Ist die deutsche Ökonomie mit ihrem Schwerpunkt bei mittleren und gehobenen Qualifikationen und Technologien dem zunehmenden internationalen Wettbewerb um Spitzenqualifikationen und -technologien hinreichend gewachsen?

(2) Dämpft die Einschränkung der Investitionen in die Bildungs-, Gesundheitsund Infrastrukturen die Bereitstellung von Humankapital, die wirtschaftliche Leistungsfähigkeit und die Nachfrage? Erhöht dies die Arbeitslosigkeit, die Prekarität und die Risiken sozialer Spannungen?

(3) Inwiefern erfordert die Notwendigkeit, die deutsche Position in der sich wandelnden internationalen Arbeitsteilung neu auszubalancieren, innen- wie außenpolitisch eine Weiterentwicklung der institutionellen und fiskalischen Steuerungsinstrumente?

Die viel diskutierten demografischen Probleme - wie Alterung, Geburtenrückgang, Zuwanderung - können nicht isoliert, sondern nur als Teil dieser sozio-ökonomischen Problematiken, etwa des steigenden Bedarfs an Gesundheits-, Bildungs- und Infrastrukturdienstleistungen, bewältigt werden.

\section{1 \\ Ein mehrdimensionales Analysemodell}

Angesichts der Herausforderungen an die politische Moderation der gesellschaftlichen Problemlagen wäre eine Gesamtlandkarte der Berufsgliederung und ihrer Dynamiken eine wichtige Orientierungshilfe. Die Entwicklung eines integrierten Analysemodells, auf das auch Prognosen gegründet werden können, steht allerdings erst an ihrem Anfang. Sie ist heute technisch leichter möglich, da hinreichend große Datenmengen und, über die vierstelligen internationalen ISCO-Kennziffern, Berufsklassifikationen für die Datenverarbeitung bereitstehen. Doch die vorliegenden Modelle für die Analyse dieser Massendaten sind entweder zu stark segmentiert oder zu undifferenziert.

Einige Berechnungen - wie die des Instituts für Arbeitsmarkt- und Berufsforschung (IAB) oder des Statistischen Bundesamtes - beschränken sich auf Trends von Einzelsegmenten der Berufsgliederung und blenden somit die Gesamtentwicklung aus. Andere Berechnungen benutzen Modelle, die zwar alle Berufe in ein Gesamtschema einfügen, aber dabei die inneren Differenzierungen der Arbeitsteilung vernachlässigen. $\mathrm{Zu}$ diesen gehört das horizontale Drei-Sektoren-Modell, das für die Dienstleistungen pauschal eine Zunahme der Erwerbstätigen von $33 \%$ auf $67 \%$, bei der Wertschöpfung von $40 \%$ auf $70 \%$ konstatiert (Geißler 2008, S. 25f.), aber auch das vertikale Erwerbsklassenmodell von Erikson/Goldthorpe (1993, S. 37, 39). Das Goldthorpe-Modell hat den Vorteil, rechentechnisch ausgereift zu sein, mischt aber alle Dimensionen (Unterschiede nach Bildung, Macht, Sektorzugehörigkeit usw.) in einer einzigen Makrodimension, der vertikalen Klassenschichtung, zusammen (was für den Zweck etwa der PISA-Studien, die Aufstiegs- und Abstiegschancen in dieser Schichtung statistisch zu messen, auch genügte). Das Modell von Bourdieu (1982), S. 195ff.) kann demgegenüber mit seinem Konzept des mehrdimensionalen sozialen Raums die widersprüchlichen Differenzierungen der gesellschaftlichen Arbeitsteilung abbilden, hat dafür aber kein rechnerisches Konzept.

Die wissenschaftlichen Diskussionen haben die neuen Differenzierungen seit den 1970er Jahren umfassend problematisiert, aber keine übersichtliche Problemlösung gefunden. Der gordische Knoten wurde von Daniel Oesch (2006) durchschlagen, der das Postulat der Mehrdimensionalität (von Bourdieu und anderen) mit der erprobten technischen Operationalisierung (von Goldthorpe und anderen) kombinierte. Er baute das Goldthorpe-Schema so um, dass eine zusammenhängende Abbildung der widersprüchlichen Differenzierungsdynamiken möglich wurde. Für jede Dynamik sieht das Modell eine eigene räumliche Differenzierungsachse vor (siehe Übersicht 1 und Tabelle 1):

Vgl. hierzu auch die eher skeptische Einschätzung der künftigen deutschen Position im Beitrag von Hübner in diesem Heft. 
- die klassische Trennlinie zwischen Unternehmern (in der rechten Spalte) und Arbeitnehmerberufen (in den drei linken Spalten),

- eine neue Einteilung nach Qualifikationsrang (vier vertikale Stufen des Bildungskapitals),

- eine horizontale Dreigliederung der Arbeitnehmerberufe nach organisatorischadministrativen, technischen und interpersonellen Arbeitslogiken,

- die Differenzierung aller Berufsgruppen nach dem Geschlecht.

Dadurch, dass die Achsen sich überschneiden, erhält Oesch eine übersichtlich strukturierte räumliche Gliederung von $17 \mathrm{Be}$ rufsgruppen oder, in der Terminologie von Max Weber, „Erwerbsklassen“, die nach zusätzlichen Kriterien weiter unterteilt werden kann. ${ }^{2}$ Die Raumstruktur ermöglicht es, Bewegungen und Differenzierungen einzeln herauszuarbeiten, die vorher durch pauschale Sammelkategorien miteinander vermischt waren. Insbesondere kann die Differenzierung der Erwerbsklassen in modernere Klassenfraktionen mit höherem Bildungskapital aufgezeigt und die Pauschalkategorie „Dienstleistungen“ in homogenere funktionale Teilgruppen zerlegt werden, die eindeutigere Entwicklungsdiagnosen ermöglichen. So wird es möglich, die Auffassung von einflussreichen Autoren wie Alain Touraine (1972) und Daniel Bell (1985), die Industriegesellschaft werde durch eine "postindustrielle Gesellschaft" oder „Wissensgesellschaft" ersetzt, auf den Prüfstand zu stellen.

Oesch nutzte sein Modell, um - entgegen der Individualisierungsthese - empirisch zu bestätigen, dass ökonomische Klassenzugehörigkeiten immer noch relevant für das politische Verhalten sind, wenn die Erwerbsklassen fein genug in homogenere Gruppen unterteilt werden. An repräsentativen Daten der Jahre 2000 und 1999 zeigte er auf, dass die Berufsgliederungen von Ländern mit verschiedenen gesellschaftspolitischen Pfaden (BRD, Schweden, Großbritannien, Schweiz) grundsätzlich ähnliche, aber nach Pfad variierende Größenproportionen haben und dass die horizontale Differenzierung mit einer bestimmten statistischen Wahrscheinlichkeit auch mit den Präferenzen für bestimmte politische Parteien korrelierte: Die Arbeitnehmer der managementnahen Berufsgruppen („organisatorische Arbeitslogik“) wählen eher konservativ und liberal, die übrigen Arbeitnehmer („technische Arbeitslogik“ und ,interpersonelle Arbeitslogik“) eher rot und grün.

Für die Dynamik der Berufsfelder hat das Modell aber erst dann einen Nutzen, wenn die Veränderungen auch im Zeitverlauf herausgearbeitet werden. Eine erste solche Längsschnittanalyse ist in Zusammenarbeit mit der Grundsatzabteilung beim Vorstand der IG Metall von einer Forschungsgruppe durchgeführt worden (Vester et al. 2009). Olaf GrohSamberg (vgl. ebd.) führte mit dem von Oesch bereitgestellten Rechenprogramm einen Probelauf mit den Daten des Soziooekonomischen Panels (SOEP) von der deutschen Vereinigung (1990) bis zum Vorabend der jüngsten Wirtschaftskrise (2007) durch. In der nachfolgenden Auswertung wurden wesentliche, miteinander verknüpfte Strukturverschiebungen klar sichtbar. Daraus sind die abgebildeten Datenübersichten ausgewählt.

Diese Datenübersichten müssen allerdings mit Bedacht gelesen werden. Sie geben Auskunft über die großen Koordinaten der berufsstrukturellen Verschiebungen, die für eine Pfaddiskussion relevant sind. Das hier benutzte SOEP umfasst eine repräsentative Stichprobe von etwa 10.000 Haushalten mit etwa 23.000 Personen, von denen gut 11.000 erwerbstätig sind, und ist damit nur für Makroanalysen tauglich. Für eine vertiefende Analyse der Berufsfelddynamiken und für ein Herunterbrechen auf Einzelentwicklungen nach Branchen, Regionen, Einkommen, Qualifikation, Geschlecht, Migrationshintergrund und Fragestellungen der Arbeitssoziologie muss dagegen das Modell weiterentwickelt und, um feinere Unterteilungen zu ermöglichen, auf erheblich größere Datensätze angewendet werden. Entsprechende Forschungen, die sich auf den jährlichen Mikrozensus des Statistischen Bundesamts mit etwa 0,82 Mio. Personen bzw. etwa 0,33 Mio. Erwerbstätigen stützen, sind in Vorbereitung.

\section{2 Strukturverschiebungen 1990-2007 im Überblick}

Abbildung 1 stellt die Entwicklung in der herkömmlichen Form eines Schichtungsdiagramms dar. Die Größenveränderungen der 17 Erwerbsklassen, die für jedes einzelne Jahr angegeben sind, verlaufen ohne plötzliche Brüche, eher stetig als schwankend und für die meisten Berufsgruppen mit mäßigen Gewinnen oder Verlusten (von 0,6 bis 2,8\%). Auffällige Ausnahmen bilden nur die Facharbeiter und Fachhandwerker, die 7,6 \% verloren, und die soziokulturellen Professionen, die 4,1 \% gewannen. ${ }^{3}$

Die abgebildeten Veränderungen können wir aber nicht dem Augenschein entsprechend als lineare Trends interpretieren, ohne die Zusammenhänge zu kennen. Diese werden erst in den räumlichen und den international vergleichenden Gesamtbildern deutlicher (vgl. Abschnitte 6 und 7 sowie Weber-Menges/ Vester in diesem Heft: Übersicht 4 und 5). Diese Gesamtbilder bestätigen sehr klar die drei langfristigen Strukturdynamiken der Höherqualifikation, der Tertiarisierung und der Feminisierung. Aber diese Entwicklungen haben nicht die Form von Trends, die linear in die Zukunft verlängert werden können und mit denen beispielsweise das Ende der Industrie oder der Facharbeit vorausgesagt werden kann. Vielmehr verlaufen die Entwicklungen der Konfliktstruktur der Gesellschaft entsprechend widersprüchlich. Der Gesamtzusammenhang der drei Dynamiken, die nachfolgend etwas näher dargestellt werden, lässt sich - thesenhaft vereinfacht - vorab wie folgt beschreiben:

(1) Durch eine anhaltende Zunahme der höheren Qualifikationen, der nur bedingt eine Bewegung zur Niedrigqualifikation gegenübersteht, erhält die Berufsgliederung die Gestalt einer Orange (Oesch 2006, S. 52) mit einer breiten, sich nach oben verschiebenden Mitte (Abschnitt 6).

(2) Im Sektor der technisch-industriellen Arbeit wirkt diesem „Upgrading“ eine Politik rigoroser Kostensenkungen entgegen, die - trotz steigender Industrieexporte die Zunahme der Beschäftigung auf der Ingenieursebene drosselt und auf allen Ebenen, besonders aber bei den Facharbeitern, sogar stark herabfährt - „polarisiertes Upgrading" (Abschnitt 7).

2 Vgl. zu diesen Diskussionen und dem Modell von Oesch mit seinen methodischen Instrumenten den Beitrag von Weber-Menges/Vester in diesem Heft

3 Die Prozentzahlen (nicht in Abb. 1 ausgewiesen) weist Übersicht 1 aus. 


\section{Abb. 1: Verschiebungen der Berufsstruktur in der BRD 1990-2007 - in \% -}

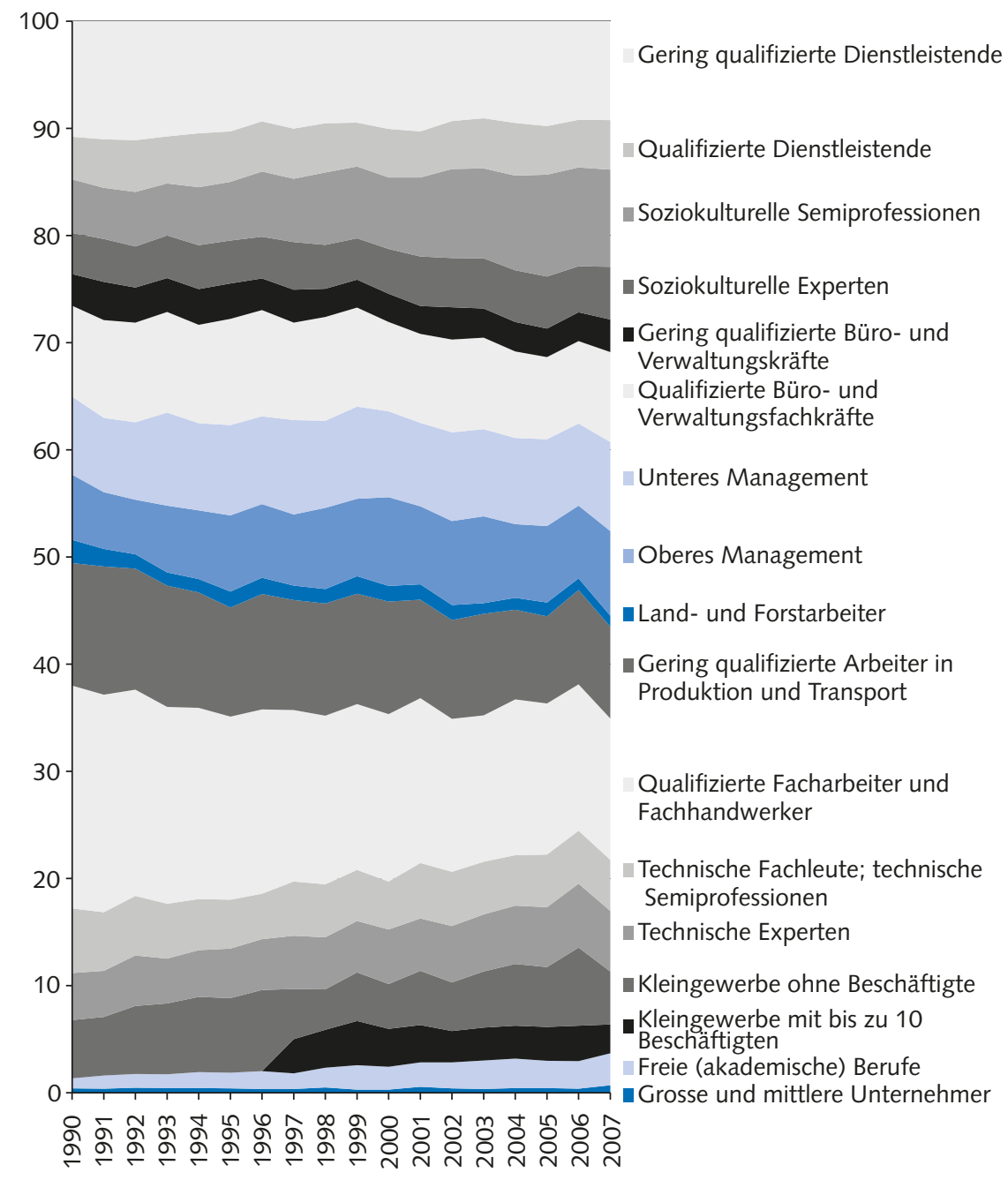

WSI MITTELUNGEN

(3) Gleichzeitig nehmen die beiden Dienstleistungssektoren anhaltend zu. Diese sind jedoch überwiegend nicht ,postindustriell“, sondern erfüllen direkt oder indirekt funktionale Bedarfe der Industrie (v. a. organisatorische Tätigkeiten und die Bereitstellung von Humankapital). Im skandinavischen Modell wurden v. a. die wohlfahrtsstaatlichen Dienstleistungen so gefördert, dass sie die anderswo freigesetzten Arbeitskräfte beschäftigen konnten (Abschnitt 8).

(4) Mit der Expansion der höher qualifizierten Berufe und der Humandienstleistungen wachsen besonders diejenigen Berufsgruppen, in denen vergleichsweise höhere Frauenanteile beschäftigt werden (Abschnitt 9).
(5) Die Entwicklungsdynamiken setzen sich dabei insgesamt nicht ungehindert um. Die drei räumlichen Bewegungen der Kompetenzsteigerung, Tertiarisierung und Feminisierung verlaufen in Deutschland langsamer und gebremster als in anderen hoch entwickelten Ländern. Die internationalen Unterschiede sind bedingt durch die verschiedenen nationalen Kräftekonstellationen bzw. Pfade der Wirtschaftsund Sozialpolitik.

Die Verschiebungen in der Berufsgliederung folgen also nicht aus abgehobenen Strukturgesetzen, sondern aus „Felddynamiken" (Bourdieu), das heißt dem Zusammenwirken und Kampf widerstreitender Markt- und Akteurskräfte, die wechselseitig voneinander abhängen und gleichzeitig in Konflikt miteinander stehen, die zu bestimmten Proportionen miteinander drängen und gleichzeitig in Schieflagen miteinander sind. Internationale Vergleiche können, wie nachfolgend gezeigt wird, für die Entwicklung dieser Proportionen zwar Hinweise geben. Jedoch sind diese nicht Maßstäbe objektiver oder richtiger Proportionen zwischen den sozialen und beruflichen Gruppen, sondern geschichtlich variabel, weil sie von den durch viele Faktoren bestimmten Entwicklungen der ökonomisch-technischen Bedingungen, der Bedarfe und Interessen der Bevölkerung und der politischen Konflikte, Entscheidungen und Kompromisse abhängen.

\section{Die Dynamik der Höher- qualifizierung}

In der vertikalen Dimension zeigen die Daten eine nachhaltige, wenn auch gebremste Bewegung in Richtung höherer Qualifikationen, der bemerkenswerterweise keine Tendenz zur Niedrigqualifikation gegenübersteht:

- Die akademischen Professionen haben sich von $15,6 \%$ auf $22,2 \%$ vermehrt.

- Die fachgeschulten Semiprofessionen haben sich von $21,5 \%$ auf $24,9 \%$ vermehrt. - Die Erwerbstätigen mit Fachlehre haben sich von 38,0 auf $31,1 \%$ vermindert.

- Die an- und ungelernten Erwerbstätigen haben sich von $27,4 \%$ auf $21,9 \%$ verringert.

Nach diesen Daten hatte die Qualifikationsverteilung schon 1990 nicht mehr die Form einer Pyramide mit einer schmalen Spitze hoher und einer breiten Basis geringerer Qualifikationen, sondern die einer Orange, in der die beiden mittleren Stufen zusammen etwa $60 \%$ einnahmen. Seitdem hat sich die breite Mitte der Orange ein Stück weit nach oben verschoben und zwar vor allem auf Kosten der Facharbeiter und Fachhandwerker.

$\mathrm{Zu}$ der Frage, wie weit diese Bewegung nach oben anhält oder durch Gegenbewegungen modifiziert wird, gibt es bisher keine eindeutigen Forschungsergebnisse. Die Diskussion stützte sich lange vornehmlich auf Länderstudien und wenig auf Vergleichsstudien. Demgegenüber bietet eine komparative Studie von Oesch/ Rodriguez (2011) weiterführende Ländervergleiche und Ursachenerklärungen an. 


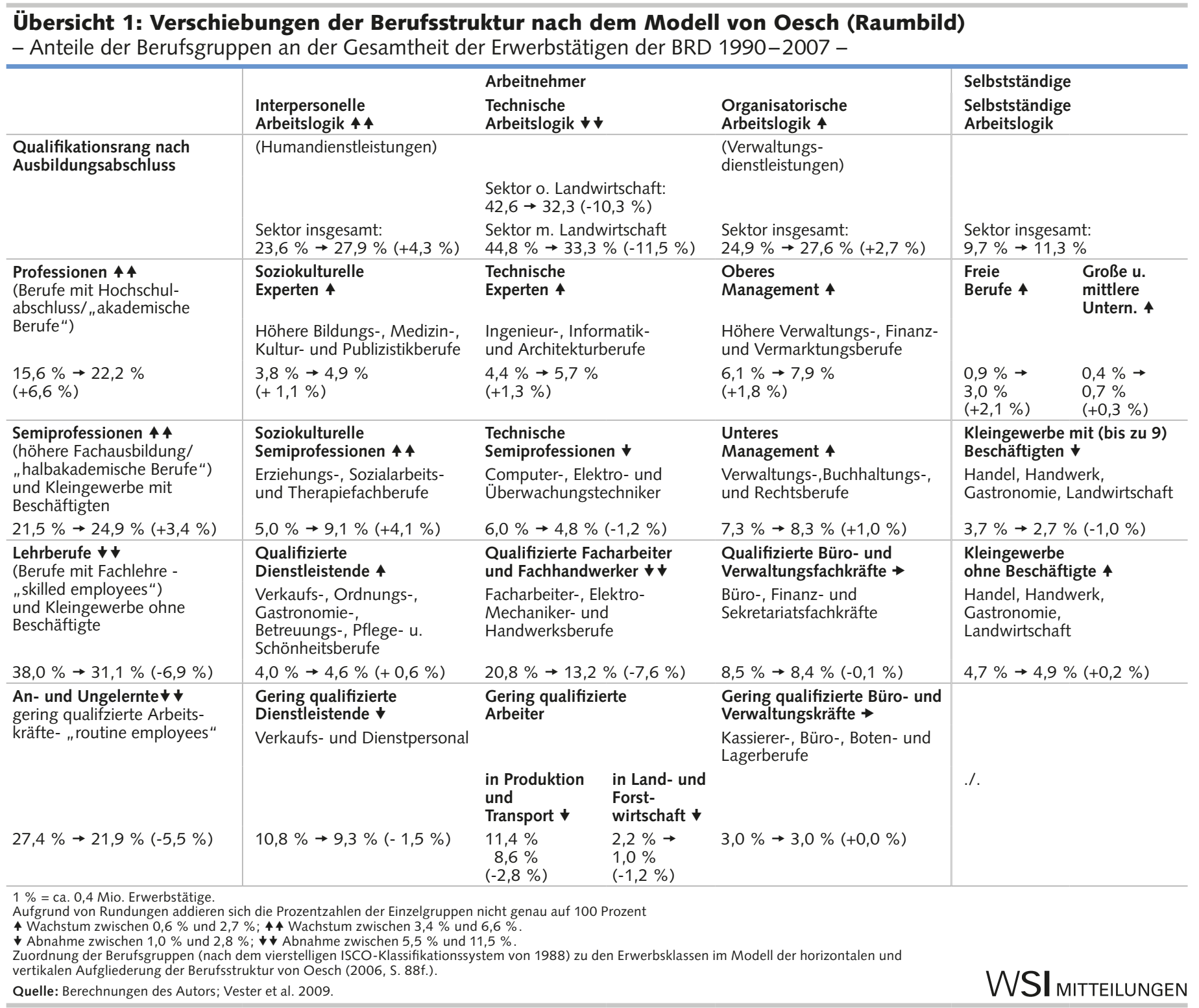

Sie benutzt allerdings nicht die mehrdimensionale Oesch-Matrix, sondern einen vertikalen Berufsgruppenvergleich ohne horizontale Sektorunterteilung. Für alle vier Vergleichsländer (Deutschland, Schweiz, Großbritannien und Spanien) stellten sie für die Periode 1990-2008 ein massives berufliches Upgrading fest, und zwar in Gestalt eines „polarisierten Upgradings", das allerdings in Deutschland erst nach 1996 und in Spanien erst nach 2002 eingesetzt hat. Dies bedeutet, dass die Beschäftigung in den mittleren Berufsgruppen stärker zurückging als in den unteren. Es wirken auf dem Arbeitsmarkt drei Faktoren zusammen: der technologische Wandel auf der Nachfrageseite, die Zunahme qualifizierter Fachausbildungen auf der Angebotsseite und die Art der Arbeitsmarktregulierung.
Das Muster eines polarisierten, die Mitte stärker ausdünnenden Upgrading wird auch von unseren Berechnungen für die Bundesrepublik bestätigt (Übersicht 1). $\mathrm{Zu}$ seiner Einordnung müssen wir etwas näher auf die Bedingungszusammenhänge eingehen.

Dass die höher qualifizierten Berufsgruppen langfristig wachsen, ist historisch nicht neu. Dies wird seit der Einführung der nationalen Statistik gemessen (Geiger 1949) und entspricht auch den klassischen Theorien und neueren Forschungen zur zunehmenden gesellschaftlichen Arbeitsteilung und Spezialisierung (Durkheim (1988); Blauner 1964; Mallet 1972; Kern/ Schumann 1984; Piore/Sabel 1989). Die entgegengesetzte Annahme eines Trends zur Dequalifizierung lässt sich eher für die mittelfristigen als für die langfristigen
Entwicklungen bestätigen. Die immer noch energisch verfolgten Politiken und Bewegungen der Vereinfachung der Arbeit beschränken sich allerdings auf bestimmte Branchen und Tätigkeiten (vor allem der technischen und organisatorischen Arbeitslogik) und deren Entwicklungszyklen, die in der Regel darein münden, dass standardisierte Arbeit schließlich in Maschinen „eingebaut" oder ausgelagert wird. Insgesamt verbirgt sich, so unsere Interpretation, hinter der Wiederkehr prekärer Erwerbsarbeit nicht eine durchgängige Abnahme der Qualifikation, sondern auch die Abwertung qualifizierter Arbeit durch niedrigere Einstufung, aber auch durch Niedriglöhne und durch unsichere Arbeitsverhältnisse.

Nicht übersehen werden darf auch, dass die statistisch festgestellte Abnah- 
me der Arbeitnehmer mit geringer und mittlerer Qualifikation einen doppelten Boden hat. Die gering qualifizierte Arbeit und - jedenfalls im Industriesektor - auch die Facharbeit nehmen weniger ab als dargestellt. Sie verschwinden in dem Maße aus der inländischen Erwerbsstatistik, in dem vor allem gering qualifizierte Arbeit in die informelle Ökonomie und vor allem industrielle Facharbeit in osteuropäische Niedriglohnländer ausgelagert wird (Abschnitt 7). Den freigesetzten Arbeitnehmern wird in Deutschland als Alternative zur Arbeitslosigkeit bisher nur der Status von prekär Beschäftigten angeboten, während auf dem skandinavischen Pfad die Entwicklung der Humandienstleistungen und der Professionen zusätzliche Arbeitsplätze geschaffen hat. Solange eine größere „Reservearmee“ des Arbeitsmarktes im Hintergrund steht, kann sie als Drohkulisse wirtschaftsliberaler Strategien dienen.

Der internationale Vergleich, den Oesch (2006) für die Zeit um 2000 errechnet hat (Abschnitt 7 sowie Weber-Menges/ Vester in diesem Heft: Übersicht 5), zeigt die Wirkung dieser Politik. Die Berufsgruppen auf der Ingenieursebene (die "technischen Professionen“) haben in der BRD trotz ihres Wachstums keine Spitzenwerte erreicht. Mit 4,5\% lagen sie 2000 um 1,4 \% bzw. 1,5 \% hinter Schweden und der Schweiz. Die technischen Semiprofessionen in der BRD haben sich sogar auf $4,9 \%$ verringert und lagen damit ebenfalls hinter denen Schwedens und der Schweiz. Die Unterschiede werden prononcierter, wenn wir diese Zahlen, die sich auf die Gesamtheit der Erwerbstätigen beziehen, für den technisch-industriellen Sektor allein betrachten. Von 100 Beschäftigten des Sektors waren im Jahre 2000 in Schweden 40 auf professionellem und semiprofessionellem Niveau beschäftigt, in Deutschland nur 27.

Demgegenüber lagen bei den interpersonellen Dienstleistungen die „Rückstände“ der BRD gegenüber Schweden eher unterhalb der obersten Qualifikationsstufe. Die BRD lag hier bei den Semiprofessionen mit 6,7 \% deutlich hinter Schweden (7,9 \%), bei den Lehrberufen mit 4,3\% sogar sehr weit hinter Schweden (9,4 \%). Auf der Ebene der Lehrberufe waren also in Schweden mehr als doppelt so viele Erwerbstätige beschäftigt. ${ }^{4}$ Bei den gering Qualifizierten war der Unterschied scheinbar geringer. Die BRD beschäftigte hier einen langsam sinkenden Prozentsatz der
Erwerbstätigen. Im Jahr 2000 umfasste er, wie in Schweden, etwa 11 \% aller Erwerbstätigen. Rechnen wir dies auf den Sektor um, der in der BRD ja kleiner ist, dann wird wieder ein deutliches Gefälle sichtbar: Von 100 Beschäftigten des Sektors waren in Deutschland 41, in Schweden aber nur 33 als gering qualifiziert eingestuft.

Insgesamt verweisen die Daten auf widersprüchliche Entwicklungen. Zum einen belegen sie ein relatives Defizit der BRD an fachlichen Berufsqualifikationen und demokratischen Bildungschancen, das aus der niedrigeren Einstufung qualifizierter Arbeitsplätze (nach erforderlichen Berufsabschlüssen) und aus einer zu geringen Förderung der Allgemein- und Berufsbildung erklärt werden kann. Auch der neue Bildungsbericht der OECD (2011) hebt hervor, „dass das Wachstum der Hochqualifizierten in Deutschland außergewöhnlich langsam verlief. In der Altersgruppe 55-64 Jahre gibt es in Deutschland 2,46 Mio. Menschen mit Tertiärabschluss, in der Altersgruppe 25-34 Jahre ist deren Zahl mit 2,48 Mio. nur geringfügig höher; demgegenüber ist die Zahl der Personen mit Tertiärabschluss in den 36 Ländern, für die entsprechende Daten vorliegen, von 39,16 auf 81,21 Mio. gestiegen und hat sich damit mehr als verdoppelt [...]“.

Zum anderen bedarf auch das Aufholen dieses Defizits langfristig einer Balance. Der vom internationalen Exportwettbewerb ausgeübte Druck auf Arbeitsverdichtung, Höherqualifikation und die Auslagerung kann durch eine extreme Flucht in das „Pilzmodell“ - übermäßige Expansion und Privilegierung der Höchstqualifikationen bei Dezimierung und Entwertung der mittleren und gehobenen Fachqualifikationen - nicht bewältigt werden.

\section{Das „polarisierte Upgrading ${ }^{\perp 1}$ in der Industrie}

Das „polarisierte Upgrading“ konzentriert sich vor allem im Sektor der technisch-industriellen Arbeit. Dabei geht es durchaus um die bisherigen Strukturen des industriellen Exportmodells der BRD. Die These aber, dass dieses Modell durch ein strukturelles Schrumpfen der industriebezogenen Arbeit seinem Ende entgegengehe, übersieht die wirklichen Gründe. Schon der Begriff „polarisiertes Upgrading“ drückt aus, dass hier zwei sehr verschiedenartige Prozesse sich überschneiden, ja in Widerspruch zueinander geraten.

Das „Upgrading“, d.h. die Höherqualifizierung der technischen Arbeit, folgt aus der Dynamik der Arbeitsteilung selber, der Steigerung des industriellen Outputs durch intelligentere Arbeit und Technologie. Wenn diese Dynamik alleine wirkte, dann müssten die Produktionssteigerungen durch nichts anderes als durch Zunahme der Beschäftigten der technischen Intelligenz in den Professionen und Semiprofessionen erzielt werden.

Die Bezeichnung „Polarisierung“ drückt aus, dass diese Dynamik durch den reinen Kostendruck konterkariert wird, durch den die Arbeit verdichtet, unter Wert eingestuft oder in Niedriglohnländer ausgelagert wird. Die „Einsparung“ von Kosten drückt auf die Beschäftigtenzahl auf allen vier Qualifikationsstufen. Sie drosselt die Beschäftigung auf den höheren Gehaltsstufen der Professionen und Semiprofessionen, am meisten bringt es aber bei den mittleren Arbeitseinkommen der facharbeiterischen Etage, weil diese zahlenmäßig am stärksten besetzt ist. ${ }^{5}$

Die Beschäftigungsrückgänge in den technisch-industriellen Berufen sind bekanntlich nicht während eines Niedergangs, sondern während eines nie dagewesenen Aufschwungs der Exportindustrien nach 1990 eingetreten. Die Supergewinne der Exportbooms wurden dabei nur sehr begrenzt durch eine höhere Teilhabe der Arbeitnehmer umverteilt. Denn der Rückgang der industriellen Beschäftigung war, wie Martin Allespach (2009, S. 278) herausgearbeitet hat, in nicht geringem Maße durch die exportfördernden Maßnahmen der Lohnkostensenkung bzw. der Arbeitsverdichtung bedingt. Die Weltmarkterfolge waren nur teilweise auf ein Produktivitätswachstum durch technische Innovationen und höhere Arbeitsqualifikationen zurückzuführen; dieses lag für Deutschland „im unteren Drittel der OECD-Länder und damit deutlich unter dem Durchschnitt. Das heißt [...], dass Deutschland seine Weltmarkterfolge in den letzten 20 Jahren

4 Die Angaben können wegen der der nationalen Unterschiede der Erwerbs- und Berufsbildungssysteme allerdings nur ungefähre Größenordnungen sein.

5 Vgl. auch den Beitrag von Hübner in diesem Heft, speziell Abschnitt 2.2 zur Problematik der Hochqualifikation im Kontext der Internationalisierung der Wertschöpfungskette. 
Tabelle 1: Wirtschaftssektoren im internationalen Vergleich

- BRD 2000/Schweden 2000/Schweiz 1999/Vereinigtes Königreich 1999 -

\begin{tabular}{|c|c|c|c|c|c|c|c|c|}
\hline \multirow[t]{2}{*}{ Größe } & \multicolumn{4}{|c|}{ Interpersonelle Dienstleistungen } & \multicolumn{4}{|c|}{ Technisch-industrielle Berufe } \\
\hline & D & S & $\mathrm{CH}$ & UK & D & 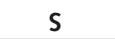 & $\mathrm{CH}$ & UK \\
\hline Sektorgröße & $26,9 \%$ & $33,1 \%$ & $26,1 \%$ & $25,7 \%$ & $36,1 \%$ & $30,2 \%$ & $31,1 \%$ & $27,3 \%$ \\
\hline \multirow[t]{2}{*}{ (Frauenanteil) } & $(61 \%)$ & $(71 \%)$ & $(53 \%)$ & $(65 \%)$ & $(15 \%)$ & $(19 \%)$ & $(18 \%)$ & $(17 \%)$ \\
\hline & \multicolumn{4}{|c|}{ Interpersonelle Dienstleistungen } & \multicolumn{4}{|c|}{ Technisch-industrielle Berufe } \\
\hline $\begin{array}{l}\text { Differenz Deutschlands zu } \\
\text { Schweden in \% }\end{array}$ & \multicolumn{4}{|c|}{$-6,2 \%$} & \multicolumn{4}{|c|}{$+5,9 \%$} \\
\hline $\begin{array}{l}\text { (Differenz im } \\
\text { Frauenanteil) }\end{array}$ & \multicolumn{4}{|c|}{$(5 \%)$} & \multicolumn{4}{|c|}{$(4 \%)$} \\
\hline \multirow[t]{2}{*}{ Zusammensetzung der Sektoren } & \multicolumn{4}{|c|}{ Interpersonelle Dienstleistungen } & \multicolumn{4}{|c|}{ Technisch-industrielle Berufe } \\
\hline & D & S & $\mathrm{CH}$ & UK & D & S & $\mathrm{CH}$ & UK \\
\hline $\begin{array}{l}\text { 1. Professionen } \\
\text { (Frauenanteil) }\end{array}$ & $\begin{array}{l}4,8 \% \\
(51 \%)\end{array}$ & $\begin{array}{l}5,1 \% \\
(51 \%)\end{array}$ & $\begin{array}{l}6,2 \% \\
(36 \%)\end{array}$ & $\begin{array}{l}4,4 \% \\
(58 \%)\end{array}$ & $\begin{array}{l}4,5 \% \\
(14 \%)\end{array}$ & $\begin{array}{l}5,9 \% \\
(25 \%)\end{array}$ & $\begin{array}{l}6,0 \% \\
(8 \%)\end{array}$ & $\begin{array}{l}3,8 \% \\
(16 \%)\end{array}$ \\
\hline 2. Semiprofessionen & $6,7 \%$ & $7,9 \%$ & $6,9 \%$ & $5,9 \%$ & $4,9 \%$ & $6,0 \%$ & $5,5 \%$ & $3,6 \%$ \\
\hline (Frauenanteil) & $(75 \%)$ & $(80 \%)$ & $(63 \%)$ & $(80 \%)$ & $(27 \%)$ & $(28 \%)$ & $(21 \%)$ & $(35 \%)$ \\
\hline 3. Lehrberufe & $4,3 \%$ & $9,4 \%$ & $3,7 \%$ & $6,1 \%$ & $13,1 \%$ & $8,6 \%$ & $9,7 \%$ & $9,9 \%$ \\
\hline (Frauenanteil) & $(47 \%)$ & $(74 \%)$ & $(50 \%)$ & $(54 \%)$ & $(6 \%)$ & $(9 \%)$ & $(7 \%)$ & $(7 \%)$ \\
\hline 4. An- und Ungelernte & $11,1 \%$ & $10,8 \%$ & $9,3 \%$ & $9,3 \%$ & $12,0 \%$ & $9,0 \%$ & $8,5 \%$ & $9,3 \%$ \\
\hline (Frauenanteil) & $(62 \%)$ & (73\%) & $(57 \%)$ & $(67 \%)$ & $(20 \%)$ & $(18 \%)$ & $(31 \%)$ & $(67 \%)$ \\
\hline
\end{tabular}

vor allem durch eine Lohnmoderation [...] errungen hat." (ebd.).

Modifiziert wird diese Entwicklung aber nicht unwesentlich durch einen dritten Faktor: das deutsche Exportmodell. Durch dieses ist die industrielle Exportquote und damit die Beschäftigung in der industriellen Produktion bedeutend höher als in anderen hochentwickelten Ländern. Die Beschäftigung im technisch-industriellen Sektor schrumpft, aber auf höherem Niveau als anderswo.

Die empirischen Größenveränderungen der technisch-industriellen Berufe spiegeln diese drei widersprüchlichen Einflüsse deutlich wider (Übersicht 1 und Tabelle 1):

(1) Der Sektor ist bis 2007 zwar spektakulär geschrumpft (-11,5\%). Aber anhand der internationalen Vergleichsdaten, die sich auf 2000 und 1999 beziehen, dürfte der Sektor doch immer noch mit großem Abstand der größte unter den Vergleichsländern sein.

(2) Hinter dem Schrumpfen steht vor allem die starke Abnahme der Facharbeiter und Fachhandwerker. Im Zeitraum von 1990 bis 2007 verminderten sich diese von $20,8 \%$ auf $13,2 \%(-7,6 \%)$, die Semiprofessionen um $1,2 \%$ und die gering Qualifizierten um 2,8\%. Hier vor allem, in der Einschnürung der facharbeiterischen Mitte, findet das "polarisierte Upgrading“ statt. Gleichwohl sind die Facharbeiter und Fachhandwerker noch die größte der 17 Erwerbsklassen in Deutschland. Im Jahre 2000 waren sie immerhin noch zwischen $3,2 \%$ und 4,5\% größer als in den Vergleichsländern.

(3) Dabei ist die Zunahme auf der obersten Etage, bei den Ingenieuren und technischen Experten durchaus „suboptimal“. Ihr Zuwachs von 4,4 \% auf 5,7 \% (+1,3\%) kompensiert bei Weitem nicht die auf den anderen Etagen verschwundenen Arbeitsplätze (-11,6 \%). Im Vergleichsjahr 2000 war ausgerechnet beim mehrfachen „Exportweltmeister" die Ingenieursetage deutlich schwächer besetzt als in Ländern wie Schweden und der Schweiz, und sie lag nur wenig vor dem Schlusslicht Großbritannien. Das Wachstum der großen Forschungszentren ist also alles andere als der Ausdruck einer allgemeinen Wachstumstendenz der akademischen Professionen.

Insgesamt ist das Schrumpfen der technisch-industriellen Berufe kein Beweis für ein objektives allgemeines Strukturgesetz, nach dem die technische Facharbeit gewissermaßen linear von der akademischen technischen Intelligenz oder den Dienstleistungen abgelöst wird. Weder der technisch-industrielle Sektor noch die Facharbeiter sind in Deutschland, wo der Export weit mehr als ein Drittel des Bruttoinlandsproduktes ausmacht, ein
„Auslaufmodell“. Es ist auch anzunehmen, dass die kostensenkende Verdichtung der Facharbeit inzwischen einen Sättigungsgrad erreicht hat und nur wenig mehr gesteigert werden kann. So ist inzwischen ein alternativer Weg der Outputsteigerungen zu beobachten: die "Umstellung“ auf einen neuen, hochqualifizierten Facharbeitertypus, der aber auch seine Rechte auf Teilhabe und Teilnahme sehr ernst nimmt. Die Differenzierung nach funktionalen Qualifikationsstufen darf nicht im Sinne einer Wertung der gesellschaftlichen Nützlichkeit von Arbeit und Berufen verstanden werden. Die kommenden Konflikte werden sich nicht nur um die Entwicklung der Höherqualifizierung, sondern auch darum drehen müssen, ob die mittleren und unteren Qualifikationsebenen unterbewertet und unterbezahlt sind.

\section{„Postindustrielle Dienst- leistungsgesellschaft" ${ }^{\mu}$ ?}

Die Frage nach der Balance von Industrie und Dienstleistungen ist nicht eine Frage des Entweder-Oder. Zum einen sind, wie dargestellt, dem Schrumpfen der technisch-industriellen Arbeit strukturelle Grenzen gesetzt. Zum anderen liefert ein erheblicher Teil der wachsenden tertiären Berufsgruppen keineswegs Dienst- 
leistungen, die „postindustrielle“ private Konsum- und Lebensbedürfnisse erfüllen, sondern unmittelbar oder mittelbar dem funktionalen Bedarf der Industriegesellschaft dienen.

Von 1990 bis 2007 sind die technischindustriellen Berufe erheblich, von 42,6\% auf $32,3 \%$, geschrumpft. Prozentual tauchen die freigesetzten $10,3 \%$ in den Dienstleistungssektoren wieder auf.

Bei den organisatorischen Berufen war das Wachstum der Erwerbstätigen von 1990 bis 2007 mit nur 2,7 \% moderat und auf die beiden oberen Stufen konzentriert. Es handelt sich um die erwerbstätigen Arbeitnehmer der Management-, Verwaltungs-, Finanz-, Vermarktungs- und Rechtsberufe, für die aufgrund zunehmender Komplexität der Bedarf gewachsen ist. Von diesen sitzt ein großer Teil in den Industrieunternehmen selbst oder in den Firmen, die ihnen zuarbeiten. Ein anderer Teil arbeitet in den öffentlichen und privaten Verwaltungs-, Versicherungs-, Rechts- und Ausbildungsinstitutionen, deren Aufwand zu den "Generalkosten“ des sekundären Sektors gehört (Geißler 2008, S. 16ff.).

Die Erwerbstätigen der interpersonellen Dienstleistungen nahmen deutlich stärker $\mathrm{zu}$, von $23,6 \%$ auf $27,9 \%(+4,3 \%)$. Es ist der einzige Sektor, in dem nicht nur die oberen Gruppen zunehmen, sondern auch die mittleren. Das größte Gewicht haben wiederum nicht die verbrauchsbezogenen Dienstleistungen, die für Konsum-, Freizeit- und Urlaubsbedürfnisse da sind. Umfangreicher sind die (eher öffentlichen) Humandienstleistungen der Bildungs-, Kultur-, Gesundheits-, Pflege-, Sozialund Ordnungsberufe. Diese sind für die steigenden Bedarfe der Ausbildung und Weiterbildung, der Gesundheit und Pflege, der Sozialdienste und der Infrastrukturen $\mathrm{da}$. Sie sind für die gesamtgesellschaftliche Entwicklung und Integration sowie die Bereitstellung von Humankapital langfristig besonders wichtig (ebd.).

In beiden Segmenten haben die produktionsbezogenen Dienstleistungen ein solches Übergewicht und eine so starke Verzahnung mit dem sekundären Sektor, dass nach Geißler (2008) nicht von einer „postindustriellen“, sondern von einer „industriellen Dienstleistungsgesellschaft" gesprochen werden muss.

Allerdings drücken die Arbeitskräfte, die nicht mehr im technisch-industriellen Sektor in der Industrie beschäftigt werden können, nachhaltig auf den Arbeitsmarkt.
Sie drängen einerseits in die Verwaltungsdienstleistungen und bleiben damit oft den Industrieunternehmen direkt oder indirekt verbunden. Andererseits drängen sie in jene Berufe, die für die Zukunftsbedarfe eines modernen Wohlfahrtsstaates wesentlich sind: die Berufe im Bildungs-, Gesundheits-, Sozial- und Kultursystem und die Berufe der akademischen technischen Intelligenz. Wie eine von dem Nobelpreisträger Stiglitz geleitete Expertengruppe formuliert hat, werden die Bildungs- und Gesundheitsberufe, ökonomisch gesehen, für die langfristige Bereitstellung von zukunftswichtigem Humankapital und die technischen Intelligenzberufe nicht zuletzt für den ökologischen Umbau gebraucht (Fitoussi/Stiglitz 2009).

Allerdings ist auch der notwendige Ausbau der Humandienstleistungen ebenfalls durch die wirtschaftsliberale Politik erheblich gebremst (Übersicht 1). Im internationalen Vergleich (Tabelle 1) bilden die Humandienstleistungen die größten Expansionspotenziale des Arbeitsmarktes. Aber in der BRD sind sie vergleichsweise wenig gewachsen. Im Jahre 2000 und lagen sie mit 26,9\% um 6,2 \% unter Schweden. Die chronische Unterfinanzierung durch den Staat drückt die Beschäftigtenzahl und die Qualität der Humandienstleistungen herab. Ein Ausbau der zukunftswichtigen Humandienstleistungen könnte dagegen, als wichtigster Hebel einer staatlichen Investitions- und Beschäftigungspolitik, die von der Industrie Freigesetzten so auffangen, dass die Arbeitslosigkeit auch langfristig und strukturell wesentlich gesenkt werden kann.

\section{Arbeitsteilung der Geschlechter: Neue Chancen und Konflikte}

Durch parallele Dynamiken des „Wertewandels" und des Erwerbssystems hat die Beteiligung der Frauen an der Erwerbsarbeit und an der Bildungsexpansion erheblich zugenommen. Allein von 1970 bis 2004 ist in Westdeutschland der Anteil der 15-65-jährigen Frauen an den Erwerbstätigen von $46 \%$ auf $65 \%$ gestiegen, in Ostdeutschland gingen 1990 sogar $92 \%$ der 25- bis 60-jährigen Frauen einer Erwerbstätigkeit nach (Geißler 2008, S. 396f.). Der internationale Vergleich der Frauenanteile zeigt gleichwohl noch eine krasse geschlechtliche Segmentierung des Arbeitsmarktes und geringere Aufstiegschancen der Frauen (Tabelle 1). Diese Ungleichheiten sind im deutschen Alleinverdienermodell der Familie stärker ausgeprägt als im schwedischen Doppelverdienermodell, das den Frauen mit dem Ausbau der Gesundheits-, Bildungs- und Sozialdienstleistungen mehr Arbeitsplätze und Weiterbildungsaufstiege anbietet (vgl. Pfau-Effinger 2000). Länder mit dem konservativen Alleinverdienermodell entlasten erwerbstätige Frauen weniger und haben daher niedrigere Geburtenraten. In Deutschland wachsen allerdings mit der Expansion der höher qualifizierten Berufe und der Humandienstleistungen ebenfalls die Berufsgruppen, in denen vergleichsweise höhere Frauenanteile beschäftigt werden, wenn auch in gebremstem Umfang (Tabelle 1).

Dies führt aber nicht dazu, dass das Ungleichgewicht zwischen männlichen und weiblichen Arbeitsmarktchancen gleichsam automatisch einem neuen "Marktgleichgewicht“ zustrebt. Auch die expandierenden Humandienstleistungen sind umkämpft. Denn in sie streben auch die jüngeren Männer aus den schrumpfenden männerdominierten Berufsgruppen.

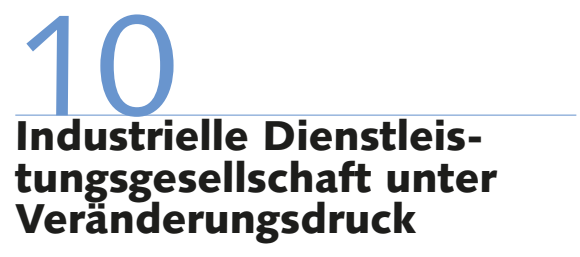

Insgesamt entspricht die reale Berufsgliederung nicht der populären These der „postindustriellen Wissensgesellschaft", die auf Touraine und Bell zurückgeht. Die Dynamik führt - nach dem Umfang der Beschäftigten wie auch der Wertschöpfung - nicht zum Ende, sondern zu einer inneren Umstrukturierung der industriebezogenen Erwerbstätigkeiten. Sie führt zum Wachsen der akademischen Professionen, aber nicht zum absoluten Übergewicht einer akademischen Wissenselite. Deren Monopol an Kompetenz und an Kommunikations- und Machtmitteln wird vielmehr durch neue Berufserfordernisse und durch die elektronischen Kommunikationsmittel immer mehr relativiert. Auch innerhalb der großen Mitte nehmen die Teilgruppen mit höheren Fachkompetenzen anhaltend und erheblich $\mathrm{zu}$ - und 
damit auch die Fähigkeiten und Forderungen von mehr Selbstbestimmung am Arbeitsplatz und mehr Mitbestimmung im Betrieb. Die Qualifikationen der Mitte erfüllen die gestiegenen funktionalen Erfordernisse der Arbeitsteilung. Sie werden nicht überflüssig.

Die Bundesrepublik wird langfristig eine „industrielle Dienstleistungsgesellschaft" (Geißler 2008) bleiben, steht aber unter Veränderungsdruck. Die Dynamiken der Arbeitsteilung wirken anhaltend und dauerhaft. Die deutsche Erwerbsstruktur reagiert auf die Erfordernisse der zunehmenden internationalen Konkurrenz durch zunehmende Hochqualifikation, industriegesellschaftliche Tertiarisierung und Einbeziehung der weiblichen Produktivkraft. Aber diese Weiterentwicklung ist nicht optimal, sondern durch die Politiken des neoliberalen Pfads gebremst. Auf der Grundlage der Längsschnittanalyse wurden vier in sich widersprüchlich strukturierte Einzeldynamiken identifiziert:

(1) „Upgrading“ auch in der Mitte: Die Höherqualifizierung hat sich erheblich und anhaltend gesteigert. Die alte Berufsgliederung hatte die Gestalt einer steilen Pyramide mit wenigen Hochqualifizierten. Inzwischen ist in der BRD fast ein Viertel der Erwerbstätigen in Berufen tätig, die ein Hochschulstudium voraussetzen. Dies ist jedoch keine absolute Tendenz, die zu einer Konzentration der Erwerbstätigen in der obersten Etage der Qualifikation, also zu einer Pilzform der Berufsgliederung, führt. Die berufliche Pyramide hat vielmehr die Gestalt einer Orange (Oesch 2006, S. 52) mit einer breiten, sich teilweise nach oben verschiebenden Mitte angenommen. Diese Mitte umfasste 2007 noch etwa $56 \%$ der Erwerbstätigen, davon rund $25 \%$ in Berufen mit Fachschulabschluss und immerhin noch $31 \%$ mit Fachlehrabschluss. Gerade auch innerhalb der letzte- ren Gruppe, auf der Etage der Facharbeiter und Fachangestellten, differenzieren sich höher spezialisierte und qualifizierte Teilgruppen heraus.

(2) „Polarisiertes Upgrading“ der industriell-technischen Qualifikationen: Im Sektor der technisch-industriellen Erwerbsarbeit sind die Beschäftigtenzahlen stark gesunken. Dies ist keineswegs das Zeichen einer abnehmenden funktionalen Bedeutung der technischen Arbeit oder der Industrie. Vielmehr wird hier das „Upgrading“ durch die polarisierenden Politiken rigoroser Kostensenkungen und Auslagerungen konterkariert. Sie drosseln - trotz steigender Industrieexporte - die Zunahme der Beschäftigung auf der Ingenieursebene und fahren sie auf den anderen Ebenen, besonders bei den Facharbeitern, sogar stark herab. Diese Politiken und die ausufernde Reglementierung stehen im Konflikt mit der Zunahme der Fachqualifikation und dem damit verbundenen Anspruch auf mehr Selbst- und Mitbestimmung.

(3) „Tertiarisierung“ mit industriegesellschaftlichem Schwerpunkt: Spiegelbildlich zur Abnahme der technisch-industriellen Erwerbsarbeit nimmt die Beschäftigung in den Dienstleistungssektoren zu. Mehr als die Hälfte dieser Beschäftigungen erfüllt jedoch nicht „postindustrielle“, sondern industrielle Bedarfe. Sie tut dies direkt (mit organisatorischen, administrativen, kommunikativen, finanziellen und rechtlichen Dienstleistungen) oder indirekt durch die Bereitstellung von Humankapital (Ausund Weiterbildung, Gesundheitswesen usw.) und durch die wohlfahrtsstaatlichen Dienste (Sozial-, Pflege-, Kultur- und Ordnungsberufe, Infrastrukturen). Die skandinavischen Modelle zeigen, dass bei hinreichender staatlicher Förderung vor allem die Humandienstleistungen so expandieren können, dass sie die in anderen
Bereichen freigesetzten Erwerbspersonen beschäftigen könnten.

(4) „Feminisierung" als Chance und Konflikt: Mit der Expansion der höher qualifizierten Berufe und der Humandienstleistungen wachsen besonders diejenigen Berufsgruppen, in denen herkömmlicherweise vergleichsweise höhere Frauenanteile beschäftigt werden. Spannungen entstehen hier dadurch, dass in der Bundesrepublik die Einkommen je Arbeitsstunde, die Wochenarbeitszeit und die Beschäftigungsstandards im Durchschnitt deutlich niedriger sind als in vielen anderen OECD-Ländern und dass zunehmend jüngere Männer in die expandierenden Berufsgruppen drängen.

Insgesamt verlaufen die drei räumlichen Bewegungen der Erwerbsgruppenstruktur in Deutschland langsamer und gebremster als in anderen hoch entwickelten Ländern. Die internationalen Unterschiede sind bedingt durch die verschiedenen nationalen Kräftekonstellationen bzw. „Pfade“ der Wirtschafts- und Sozialpolitik. Der herrschende neoliberale Pfad kommt von zwei Seiten unter Druck. Zum einen haben parallel zur Höherqualifikation, Tertiarisierung und Feminisierung der Arbeit auch die Kompetenzen und Ansprüche zu mehr Selbstbestimmung und Mitbestimmung in Betrieb und Gesellschaft zugenommen (vgl. Vester et al. 2001, 2007). Dem entspricht die hohe Unzufriedenheit der Arbeitnehmer und der Bevölkerung mit alten und neuen Privilegienstrukturen. Zum anderen wächst die Gefahr einer neuen weltweiten Rezession. Diese ist eine Folge der innerwirtschaftlichen und internationalen Ungleichgewichte, die durch die von oben verordnete Austeritätspolitik in Europa verstärkt werden und daher in Form der Eurokrise und erneut nachlassender Exportnachfrage auf die Bundesrepublik zurückkommen. 


\section{LITERATUR}

Allespach, M. (2009): Gewerkschaftliche Eckpunkte einer aktiven Industriepolitik, in: WSI-Mitteilungen 62 (5), S. 277-280

Bell, D. (1985) [1975]: Die nachindustrielle Gesellschaft, Frankfurt a. M./ New York

Blauner, R. (1964): Alienation and Freedom - The Factory Worker and His Industry, Chicago/London

Bourdieu, P. (1982) [1979]: Die feinen Unterschiede, Frankfurt a. M.

Durkheim, E. (1988) [1893/1902]: Über soziale Arbeitsteilung, Frankfurt a. $M$.

Erikson, R./Goldthorpe, J. H. (1993): The Constant Flux, Oxford Fitoussi, J.-P./Stiglitz, J. (2009): The Ways Out of the Crisis and the Building of a more Cohesive World, Document de travail OFCE (Observatoire Francais des Conjonctures Économiques) 2009-17, http://www. ofce.sciences-po.fr/pdf/dtravail/WP2009-17.pdf

Geiger, Th. (1949): Die Klassengesellschaft im Schmelztiegel, Köln/ Hagen

Geißler, R. (2008): Die Sozialstruktur Deutschlands, Wiesbaden GraßI, H. (2000): Strukturwandel der Arbeitsteilung. Globalisierung, Tertiarisierung und Feminisierung in der Wohlfahrtsproduktion, Konstanz

Kern, H./Schumann, M. (1984): Das Ende der Arbeitsteilung? Rationalisierung in der industriellen Produktion: Bestandsaufnahme, Trendbestimmung, München

Kocka, J. (1980): The study of social mobility and the formation of the working class in the 19th century, in: Le Mouvement Social (111), S. $97-118$
Mallet, S. (1972) [1963]: Die neue Arbeiterklasse, Neuwied/Berlin OECD (2011): Bildung auf einen Blick 2011. OECD-Indikatoren, Bielefeld Oesch, D. (2006): Redrawing the Class Map. Stratification and Institutions in Britain, Germany, Sweden and Switzerland, Basingstoke Oesch, D./Rodriguez, J. (2011): Upgrading or Polarization? Occupational Change in Britain, Germany, Spain and Switzerland, 1990-2008, in: Socio-Economic Review 9 (3), S. 503-532

Pfau-Effinger, B. (2000): Kultur und Frauenerwerbstätigkeit in Europa. Theorie und Empirie des internationalen Vergleichs, Opladen

Piore, M. J./Sabel, Ch. F. (1989) [1984]: Das Ende der Massenproduktion: Studie zur Requalifizierung der Arbeit und die Rückkehr der Ökonomie in die Gesellschaft, Berlin

Touraine, A. (1972) [1969]: Die Postindustrielle Gesellschaft, Frankfurt a. $M$.

Vester, M./Gardemin, D./Groh-Samberg, O. (2009): Zwischenergebnisse der Berufsfeldanalyse nach Oesch auf der Grundlage des Sozioökonomischen Panels (SOEP) 1990-2007 (Forschungsbericht), Hannover

Vester, M./Teiwes-Kügler, Chr./Lange-Vester, A. (2007): Die neuen Arbeitnehmer. Zunehmende Kompetenzen - wachsende Unsicherheit, Hamburg

Vester, M./von Oertzen, P./Geiling, H./Hermann, Th./Müller, D. (2001): Soziale Milieus im gesellschaftlichen Strukturwandel. Zwischen Integration und Ausgrenzung, Frankfurt a. M. 